\title{
Long Term Variation of Solar Corona from SOHO/EIT Observations
}

\author{
Jie Zhang ${ }^{1}$ and Mukul R. Kundu ${ }^{2}$ \\ ${ }^{1}$ School of Computational Sciences, George Mason University, 4400 University Dr., Fairfax, VA \\ 22030, USA email: jiez@scs.gmu.edu \\ ${ }^{2}$ Astronomy Department, University of Maryland, College Park, MD 20742
}

\begin{abstract}
.
We present the long term variation of solar corona based on SOHO/EIT observations from 1996 to 2004. EIT provides diagnostics of bulk corona in three channels with overlapping temperature range from $0.5 \mathrm{MK}$ to $2.7 \mathrm{MK}$ and with high spatial resolution. We find that the coronal emission measure increases by a factor of 4 from $2.0 \times 10^{27} \mathrm{~cm}^{-5}$ at the solar minimum to $8.0 \times 10^{27} \mathrm{~cm}^{-5}$ at the solar maximum. In the meantime, the overall temperature of the corona increases from 1.3 MK to $1.7 \mathrm{MK}$
\end{abstract}

\section{Introduction}

The Sun's corona, at temperatures more than 1 million Kelvin, is the primary source of EUV irradiance that are responsible for the creation and controlling of the Earth's ionosphere. The corona is known to be driven by the magnetic cycle of the Sun, though its exact heating mechanism remains to be identified. In this paper, we present a quantitative study on the long term variation of the corona on the solar cycle time scale, in terms of both its temperature and emission measure (or density). The results may help understanding the origin of the solar corona and its effect on the Earth's ionosphere.

\section{Observations and Results}

This study is based on the observations of Extreme-ultraviolet Imaging Telescope (EIT, Delaboudinière et al. 1995) on board the Solar and Heliospheric Observatory (SOHO) spacecraft launched in 1995 December. EIT images the whole Sun with a spatial resolution of 5.2" in four EUV passbands, centered at $171 \AA, 195 \AA, 284$ A and $304 \AA$, respectively. The first three passbands, which are selected to observe coronal emission lines of $\mathrm{Fe} \mathrm{XI} / \mathrm{X}, \mathrm{Fe} \mathrm{XII}$ and Fe XV, are sensitive to narrow coronal temperature ranges peaked at $0.87,1.32$ and $2.00 \mathrm{MK}$, respectively. EIT has been carrying out the so called synoptic observation plan, with which a set of 4 snapshot solar images, each of the four passbands, are taken within a short period (e.g., $30 \mathrm{~min}$ ); it is executed every six hours.

For the purpose of this study, we have selected one set of synoptic observation every month, 95 sets in total from 1996 January to 2004 March (except for a few months during SOHO interruption in 1998). We use the so-called two-temperature model (Zhang et al. 1999) to derive the temperature (Te) and emission measure (EM) map of the corona. This method is an enhanced version of the traditional image-ratio method to calculate Te and EM. Traditional method is to use images of two passbands to calculate a single Te and EM, e.g., using $171 \AA$ and $195 \AA$ only. However, with three coronal observations of overlapping temperatures from EIT, a single temperature method will only reproduce the emission measure within the two passbands used, which under-estimates the total emission measure. This problem is solved by assuming a two-temperature component, $T_{L}$ (Lower Temperature) and $T_{H}$ (Higher Temperature), for the coronal atmosphere 

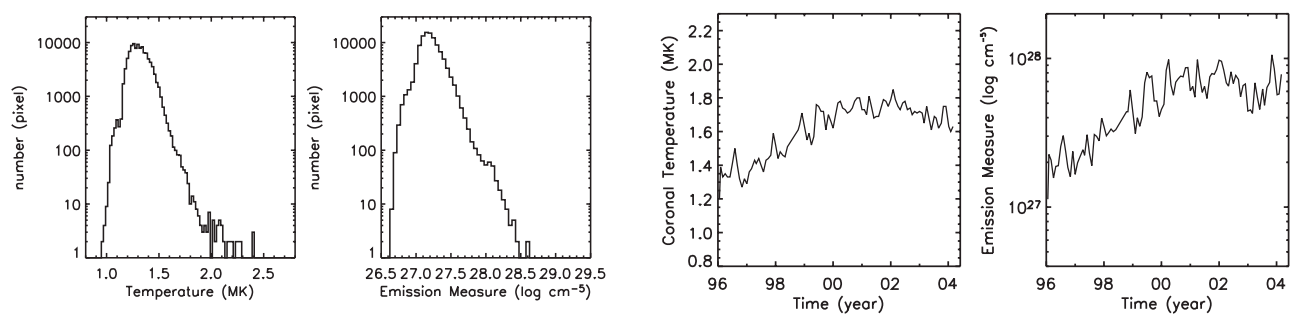

Figure 1. Left Panels: Derived coronal temperature (left) and emission measure distribution (right) on 1996 May 01. Right panels: Overall coronal temperature (left) and emission measure variation during the solar cycle 23

integrated along the line of sight for each pixel. A unique solution is achieved by adding one constraint that is to seek minimal $T_{H} / T_{L}$ ratio. The final EM result will be the sum of the two components, while the final temperature is the temperature weighted by emission measures: $\mathrm{Te}=\left(T_{L} \times E M_{L}+T_{H} \times E M_{H}\right) /\left(E M_{L}+E M_{H}\right)$.

In Figure 1 (left panels), we show the solution for the observations on 1996 May 01. The left panel shows the histogram distribution of coronal temperature for all pixels within solar disk. In this typical solar minimum day, coronal temperature ranges from about $0.95 \mathrm{MK}$ for quiet Sun regions to $2.3 \mathrm{MK}$ for small active regions; the peak temperature is about $1.26 \mathrm{MK}$ and the average temperature is about $1.33 \mathrm{MK}$. The right panel shows the distribution of emission measure, which ranges from $4.0 \times 10^{26} \mathrm{~cm}^{-5}$ in coronal hole to $4.0 \times 10^{28} \mathrm{~cm}^{-5}$ in small active regions, with a peak value at $1.4 \times 10^{27} \mathrm{~cm}^{-5}$ and an average value at $1.9 \times 10^{27} \mathrm{~cm}^{-5}$.

The solar cycle variations of Te and EM from 1996 to 2004 are shown in Figure 1(right panels). Since the solution for one set of observations has detailed pixel by pixel value, we simply average the whole disk pixels to get a single temperature and emission measure, which shall represent the overall property of the corona for that particular moment. There is a clear trend that coronal temperatures steadily increases from $1.3 \mathrm{MK}$ at the solar minimum to about $1.7 \mathrm{MK}$ at the solar maximum; the temperature starts to decrease after 2002. On the other hand, overall coronal emission measure increases by a factor of 4 from $2.0 \times 10^{27} \mathrm{~cm}^{-5}$ at the solar minimum to $8.0 \times 10^{27} \mathrm{~cm}^{-5}$ at the solar maximum.

\section{Discussion}

The calculated emission measure subjects to the selection of elemental abundance. In this study, we use the photospheric abundance, which may under-estimate Fe and other low FIP elemental abundance in the corona (e.g., Meyer 1985). If coronal abundance is adopted, the absolute value of emission measure may decrease by a factor of 2 to 4 . Nevertheless, the relative change of temperature and emission measure with solar cycle will largely remain the same.

\section{Acknowledgements}

J. Zhang is supported by NASA grants NAG5-11874 and NSF SHINE grant ATM0203226 .

\section{References}

Delaboudinière, J.-P., et al. 1995 Sol. Phys. 162, 291

Meyer, J.-P., 1985 ApJS 57, 173

Zhang, J., White, S.M., \& Kundu, M.R. 1999 ApJ 527, 977 


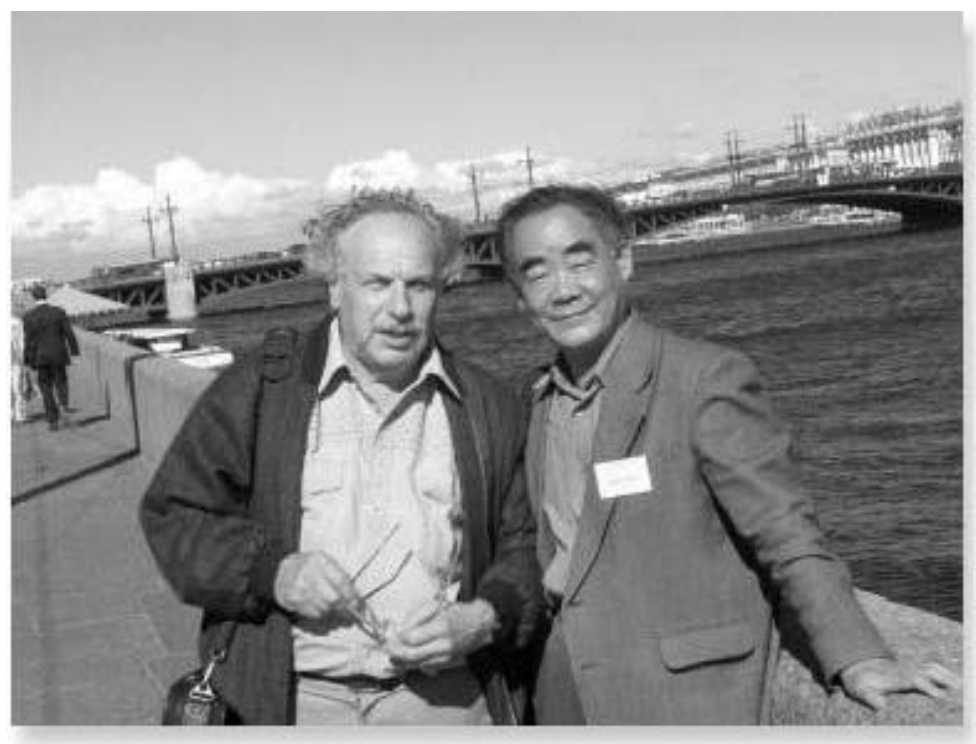

Valeri Skomorovski (Russia) and Jingxiu Wang (China).

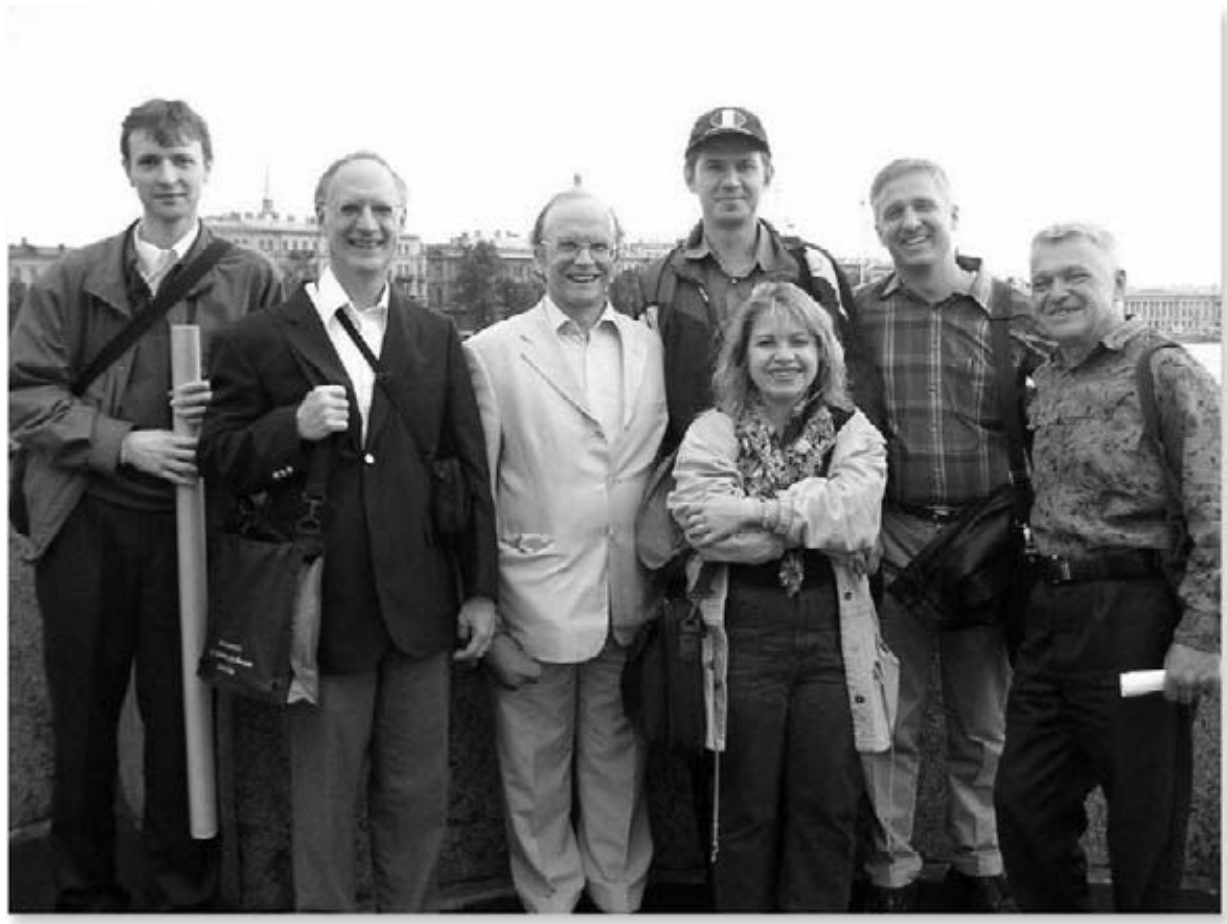

Maxim Kramar (Germany), David Webb (USA), Eric Priest (UK), Barbara Thompson (USA), Andrei Zhukov (Belgium), David Byers (USA), Igor Veselovsky (Russia). 


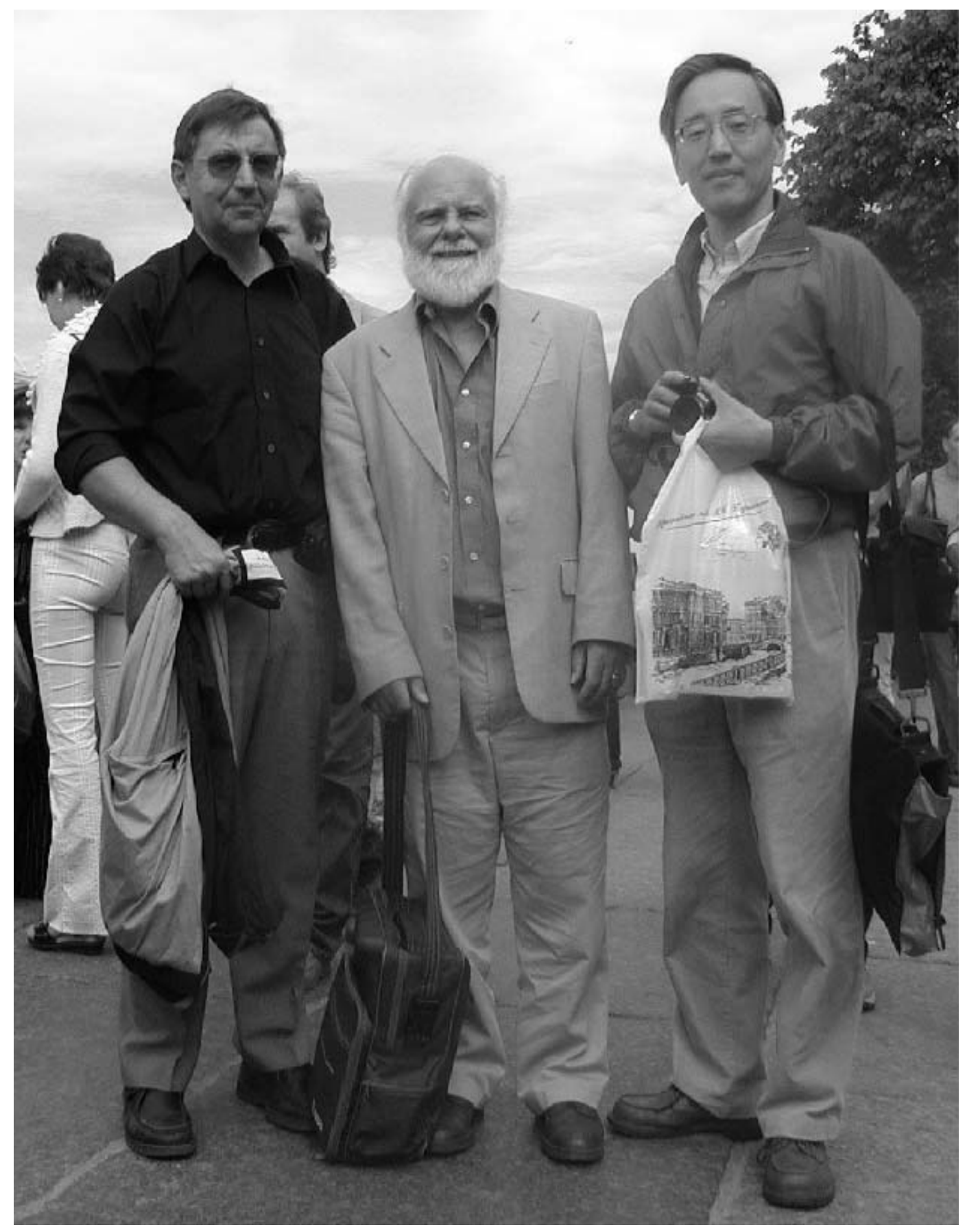

Alexander Kosovichev (USA), Douglas Gough (UK), Hiromoto Shibahashi (Japan). 